\title{
Sputtering Yields of Pure and Helium-Implanted Tungsten Under Fusion-Relevant Conditions Calculated Using Molecular Dynamics
}

\author{
Francesco Ferroni $^{\mathrm{a}}$, Karl D. Hammond ${ }^{\mathrm{b}, 1}$, Brian D. Wirth ${ }^{\mathrm{b}, \mathrm{c}, *}$ \\ ${ }^{a}$ Department of Materials, University of Oxford, Parks Road, Oxford, OX1 3PH (UK) \\ ${ }^{b}$ Department of Nuclear Engineering, University of Tennessee, Knoxville, TN 37996 (USA) \\ ${ }^{c}$ P.O. Box 2008, MS-6003, Oak Ridge National Laboratory, Oak Ridge, TN 37831 (USA)
}

\begin{abstract}
This study uses atomistic simulations to calculate the sputtering yield of tungsten in the presence and absence of near-surface helium clusters. We find that near-surface helium in the concentrations tested (consistent with $100 \mathrm{eV}$ implantation fluences of 1.0 and $2.2 \times 10^{19} \mathrm{He} \cdot \mathrm{m}^{-2}$ at a flux on the order of $10^{25} \mathrm{He} \cdot \mathrm{m}^{-2} \mathrm{~s}^{-1}$ ) has a negligible effect on the sputtering yield, suggesting that any changes in sputtering that might be observed experimentally are due either to much higher near-surface concentrations of helium or due to other structural changes in the tungsten that would occur only at much higher helium fluences than we are able to probe using molecular dynamics simulation. We do find a dependence on surface orientation and incident helium energy: comparing $\left\{\begin{array}{lll}0 & 0 & 1\end{array}\right\},\left\{\begin{array}{lll}1 & 1 & 0\end{array}\right\}$, and $\left\{\begin{array}{lll}1 & 1 & 1\end{array}\right\}$ surfaces, we find that $\left\{\begin{array}{llll}1 & 1 & 0\end{array}\right\}$ surfaces at high energy show the most sputtering, while $\{111\}$ surfaces at low energy show the least. We attribute these differences in surface orientation to the more closely-packed nature of $\left\{\begin{array}{lll}1 & 1 & 0\end{array}\right\}$ surfaces compared to the other two orientations.
\end{abstract}

\footnotetext{
*Author to whom correspondence should be addressed.

Email address: bdwirth@utk.edu (Brian D. Wirth)

${ }^{1}$ Present address: Department of Chemical Engineering, University of Missouri, Columbia, MO 65211 (USA)
} 


\section{Introduction}

Tungsten is the primary candidate material for the divertor in future nuclear fusion reactors such as ITER [1]. While one reason for choosing tungsten is its lack of chemical sputtering, physical sputtering is possible at sufficiently high incident energies. For helium, the main product of fusion reactions, the threshold energy to sputter tungsten is approximately $105-110 \mathrm{eV}$, which is in the range of expected energies of particles in the baffle region of the ITER divertor [2]. Over time, the cumulative atomic-scale processes of physical sputtering can produce erosion and shorten the divertor lifetime. Current expectations are that physical erosion should be negligible in the detached-divertor regime, with expected ion energies of $\approx 15 \mathrm{cV}[2]$. However, components such as those near the baffle region of the divertor, which are near the interface of the hot scrape-off layer (SOL) of the plasma edge, may be exposed to particles with energies significantly higher than $100 \mathrm{eV}$, creating physical sputtering. Expected ion energies at the midplane are expected to be approximately $500 \mathrm{eV} \mathrm{[2].} \mathrm{Consequently,}$ it is of interest to quantify the sputtering yields caused by helium at energies relevant to ITER, and to determine whether the sputtering yield will change significantly as the device ages and/or is modified by implanted gas atoms.

Experiments in linear plasma devices demonstrate that a very high density of nanometre-sized helium bubbles form in near-surface layers of the tungsten matrix [3-6]. This has been confirmed to occur on much smaller time and length scales in molecular dynamics simulations [7-10]. Therefore, it is also necessary to understand whether sputtering rates change with increasing levels of helium retention in the material.

This study compares the sputtering yield of tungsten due to helium ions in the presence and absence of near-surface helium atoms using molecular dynamics. Specifically, we determine whether the near-surface "layer" of substitutional helium atoms and small helium atom clusters observed in our previously reported atomistic simulations [8] have an effect on the sputtering yicld. We find that helium in the concentrations so far observed in such simulations has a 
negligible effect on the sputtering yield, suggesting that any changes in sputtering are due either to much higher near-surface concentrations of helium, or to other structural changes in the tungsten that would occur only at much higher fluence.

\section{Procedure}

Molecular dynamics (MD) simulations were performed using LAMMPS [11]. The interatomic potentials used were the Ackland Thetford modification of the Finnis-Sinclair $N$-body potential for tungsten [12, 13], as modified at small interatomic separations for sputtering and cascade simulations by Juslin and Wirth [14]; the tungsten-helium potential of Juslin et al. [14]; and Beck's potential for He-He interactions $[15,16]$ as modified at small interatomic separations by Morishita et al. [17].

Molecular dynamics simulations of sputtering were performed on pure tungsten surfaces and helium-implanted tungsten surfaces. Helium-implanted simulations were bombarded with helium ions with energies between $200 \mathrm{eV}$ and $500 \mathrm{eV}$. The set-up of each kind of simulation is detailed in the following subsections.

It should be noted that molecular dynamics simulations neglect electronic stopping, which can be a significant fraction of the energy loss. Electronic stopping is small at low ion energies, and neglecting it is a good approximation for heavy ions such as iron or tungsten; however, helium is light enough that nuclear stopping is also small, so the net effect is that electronic stopping cannot be neglected without introducing some error into the simulation. For example, TRIM calculations using 10,000 helium atoms impinging on a tungsten surface suggest that 56 percent of the stopping power comes from electronic stopping (ionization) for $200 \mathrm{eV}$ incident helium, while 59 percent comes from electronic stopping for $500 \mathrm{eV}$ incident helium. As such, things like sputtering yields and depth distributions as calculated from molecular dynamics simulations should be interpreted with this in mind. In particular, it is fine to make comparisons of the 
relative differences in depth across different surface orientations, but the actual depth distributions will be too "deep" due to neglect of electronic stopping.

\subsection{Set-Up for Pure Tungsten Case}

In the case of pure tungsten, three different surface orientations were used, namely (001), (011), and (111). The $x$ - and $y$-directions of the simulation box had periodic boundary conditions, while the $z$-direction was a free surface (with one surface exposed to the plasma). The depth of the simulation cell was approximately $44.5 \mathrm{~nm}$ in the $z$-direction. The height and width varied from 3.8 to $5.4 \mathrm{~nm}$ in the $x$ - and $y$-directions, depending on the surface orientation. The system was subsequently thermalised to $933 \mathrm{~K}$ using a Nosé-Hoover thermostat [ 18 20] with a time constant of $0.05 \mathrm{ps}$ and a time step of $0.5 \mathrm{fs}$, and the system state was saved in a restart file.

A sputtering simulation consists of reading the state from the aforementioned restart file, then introducing a helium atom above the free surface with the appropriate kinetic energy, aimed normal to the surface. Each simulation was then time-integrated using a variable time step for $5 \mathrm{ps}$, which was observed to be sufficient for helium even at high energies to thermalise and for any sputtered tungsten atoms to be ejected from the free surface. The time step during the sputtering simulation was chosen to be in the range $1.0 \times 10^{-5}$ fs to $0.5 \mathrm{fs}$, with the step size re-sampled every ten steps such that no atom moved more than $0.01 \AA$ during the first of the ten steps. At the end of each sputtering simulation, any atoms more than two lattice units above the free surface were considered to have sputtered. Owing to the rarity of a sputtering event, the simulation was repeated between 4000 and 8000 times to ensure satisfactory statistics. Each of these 4000-8000 simulations introduced the helium atom at a different random position in the $x-y$ plane above the free surface. The sample sizes for each surface orientation in the pure tungsten cases are shown in Table 1.

\subsection{Set-Up for Helium Pre-Implanted Tungsten Case}

In the case of tungsten pre-implanted with $100 \mathrm{eV}$ helium, a large box $(50 \times$ $50 \times 23 \mathrm{~nm})$ of pure tungsten was created with a (111) surface orientation. 
Table 1: Number of individual, independent sputtering simulations on pure tungsten with varying surface orientations and helium energies. All simulations performed at $933 \mathrm{~K}$.

\begin{tabular}{lllll}
\hline & \multicolumn{4}{c}{ Energy $(\mathrm{eV})$} \\
\cline { 2 - 5 } Surface Orientation & 200 & 300 & 400 & 500 \\
\hline$\left(\begin{array}{llll}0 & 0 & 1\end{array}\right)$ & 8000 & 8000 & 8000 & 8000 \\
$(011)$ & 4000 & 4000 & 4000 & 4000 \\
$(111)$ & 4000 & 6000 & 6000 & 6000 \\
\hline
\end{tabular}

This was cumulatively implanted with thermalised helium introduced at depths sampled from a cumulative distribution function associated with an incident ion energy of $100 \mathrm{eV}$ at a temperature of $933 \mathrm{~K}$ and a flux of $7.9 \times 10^{25} \mathrm{He} \cdot \mathrm{m}^{-2} \mathrm{~s}^{-1}$. The state of this simulation was sampled at fluences of $1.0 \times 10^{19} \mathrm{He} \cdot \mathrm{m}^{-2}$ or $2.2 \times 10^{19} \mathrm{He} \cdot \mathrm{m}^{-2}$. In this simulation, helium very close to the surface causes trap mutation, creating an adatom-vacancy pair and resulting in a "layer" with locally high helium concentration consisting of substitutional helium as well as small helium-vacancy clusters, as shown in Figure 1. For further details on how this simulation was carried out, please refer to Ref. 8.

For the purpose of this study, a smaller, more tractable simulation was created by sampling a $5.2 \times 5.2 \times 23.1 \mathrm{~nm}$ block from the upper corner of the large simulation. This block of tungsten and helium was then thermalised to $933 \mathrm{~K}$ $\left(660^{\circ} \mathrm{C}\right)$ using the same thermostat as in the pure tungsten case, and the state was subsequently saved in a restart file. An identical procedure to the case of pure tungsten was used to estimate the sputtering rate, with the only difference being the starting state. The numbers of both tungsten and helium atoms sputtered from the free surface were tracked for each simulation, and each simulation was repeated between 4000 and 6000 times using a different $x-y$ position, as in the pure tungsten case.

\section{Sputtering of Pure Tungsten}

\subsection{Sputtering Yield}

The predicted sputtering yields of tungsten from helium irradiation as a function of surface orientation and helium energies are shown in Figure 2. The results show that $\mathrm{W}\left\{\begin{array}{lll}1 & 1 & 0\end{array}\right\}$ surfaces have a significantly higher sputtering yield 


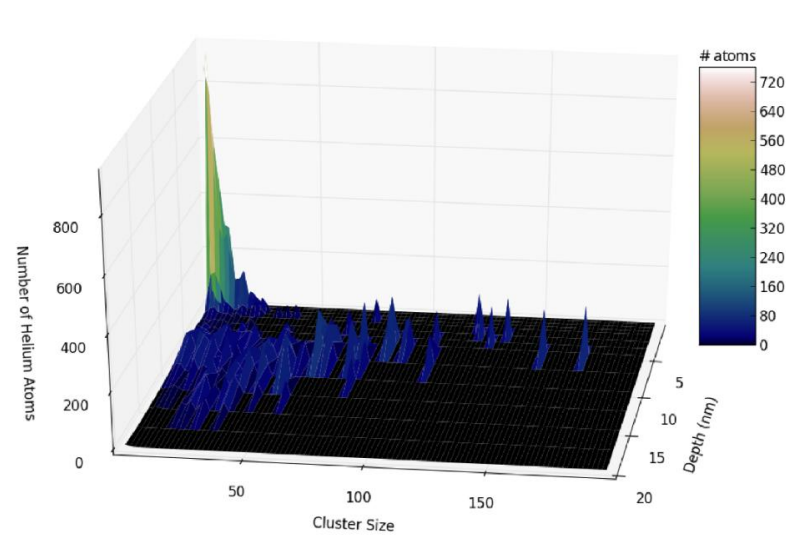

(a)

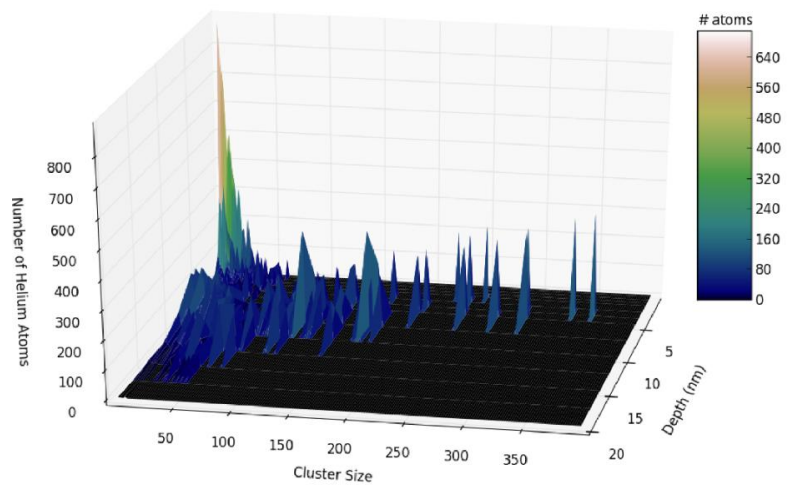

(c)

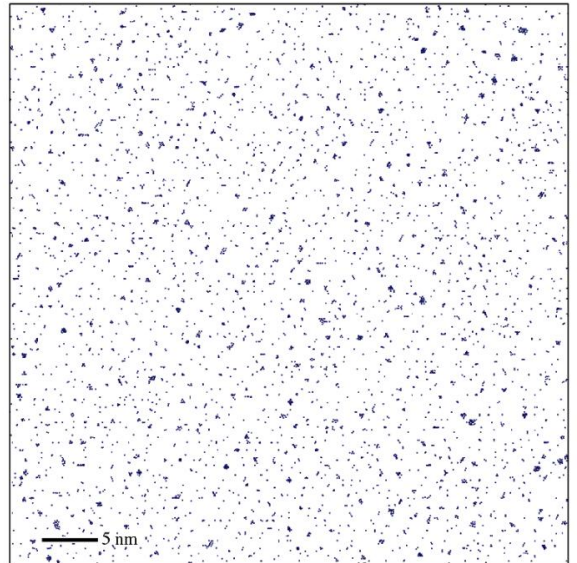

(b)

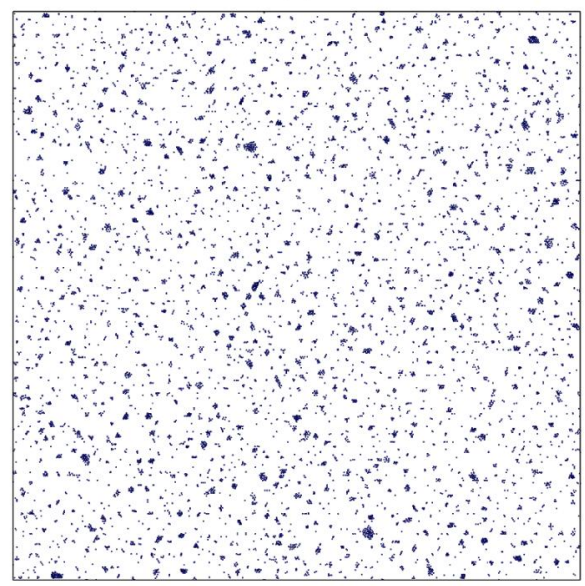

(d)

Figure 1: (a),(c): Size distributions of helium clusters for helium implantation fluences of $1.0 \times 10^{19} \mathrm{Hc} \cdot \mathrm{m}^{-2}$ and $2.2 \times 10^{19} \mathrm{He} \cdot \mathrm{m}^{-2}$, respectively. (b),(d): Frontal vicws of helium atoms in the starting configurations for our pre-implanted tungsten sputtering simulations (for 1.0 and $2.2 \times 10^{19} \mathrm{He} \cdot \mathrm{m}^{-2}$, respectively). All visible atoms in parts (b) and (d) are fewer than four lattice units below the (111)-oriented surface. 


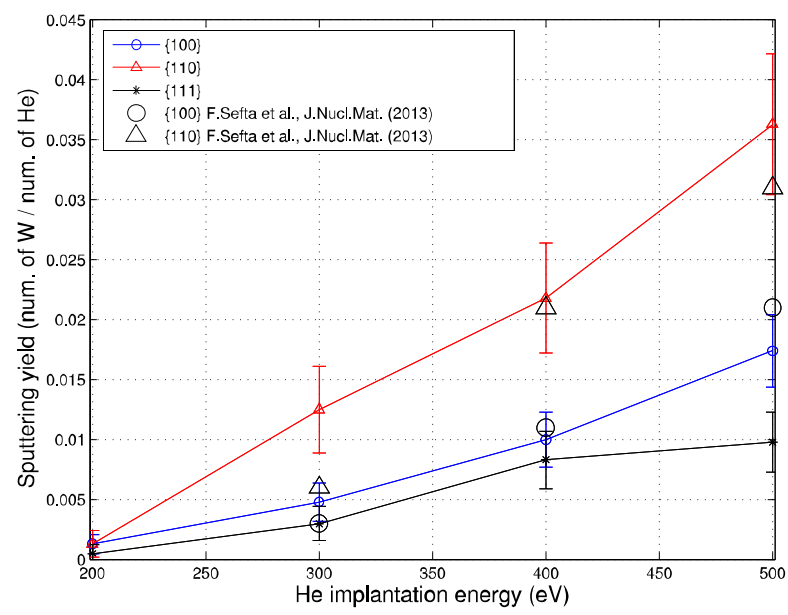

Figure 2: Sputtering yields of pure, single-crystal tungsten as a result of helium irradiation as a function of incident energy for three different surface orientations. Error bars correspond to $95 \%$ confidence intervals. Data for $\left\{\begin{array}{lll}1 & 0 & 0\end{array}\right\}$ and $\left\{\begin{array}{lll}1 & 1 & 0\end{array}\right\}$ surfaces in the absence of helium from Sefta et al. [21] are shown for comparison.

than $\mathrm{W}\{100\}$ or $\mathrm{W}\{111\}$ surfaces. This is qualitatively reasonable, because $\{110\}$ surfaces are the most closely-packed of the three, and hence most likely to produce a direct helium-tungsten collision. This observation is consistent with experimental results for single-crystal BCC metals [22], which showed the same difference between $\operatorname{Mo}\{100\}$ and Mo\{110\} surfaces. $\{111\}$ surfaces are the least closely-packed of the three orientations studied, and also show the lowest sputtering yields. Voitsenya and coworkers [23] came to the same conclusions for largely the same reasons based on experimental sputtering results. This is also supported by the respective depth distributions of helium at the end of each sputtering simulation, as shown in Figure 3. The sputtering yields also increase with increasing incident energy, but the sputtering yield begins to level off for the (111) surface at $500 \mathrm{eV}$. This behaviour is consistent with the behaviour seen experimentally [24], but it should be noted that our previous simulations [21] showed no such decrease even at energies as high as $1000 \mathrm{eV}$, suggesting this observation may be due at least in part to sampling error. We emphasize again that these simulations neglect electronic stopping, which is a significant source 
Table 2: Percentage of incident helium atoms that remain below a tungsten surface without reflecting or rapidly escaping as a function of helium encrgy and surface oricntation.

\begin{tabular}{lllll}
\hline & \multicolumn{4}{c}{ Energy $(\mathrm{eV})$} \\
\cline { 2 - 5 } Surface Orientation & 200 & 300 & 400 & 500 \\
\hline$\left(\begin{array}{llll}1 & 0 & 0\end{array}\right)$ & $53.4 \%$ & $59.7 \%$ & $62.0 \%$ & $62.2 \%$ \\
$(110)$ & $40.0 \%$ & $43.2 \%$ & $47.5 \%$ & $48.9 \%$ \\
$(111)$ & $63.3 \%$ & $67.2 \%$ & $68.9 \%$ & $71.0 \%$ \\
\hline
\end{tabular}

of error, particularly at high energies. In particular, this is believed to be the primary cause of the discrepancy between the sputtering yields calculated here and by Sefta et al. [21] compared to those measured experimentally, as shown in Figure 4.

\subsection{Depth Distributions}

The incident energy of the helium atoms and the orientation of the free surface affect the fraction of helium atoms which embed in the metal. The fraction of atoms that do not reflect or rapidly escape into the gas phase are shown below in Table 2 .

The depth distributions of helium after thermalisation are shown in Figure 3. Unsurprisingly, the mean depth increases as a function of incident energy. The depth is also highly dependent on the surface orientation, consistent with our previous findings for $100 \mathrm{cV}$ helium [8] and with the recently published results of Borovikov [25]. In the (111) case, helium atoms are embedded substantially deeper than in the $(100)$ case, presumably due to atomic channelling.

We would like to re-emphasize at this point that electronic stopping is neglected in these simulations. As such, it is perhaps inappropriate to consider the absolute depth distributions at face value. It is, however, acceptable to compare relative depths between different surface orientations, as electronic stopping is neglected similarly in such situations.

\section{Comparison between Pure and Pre-Implanted Tungsten}

\subsection{Tungsten Sputtering}

There appears to be no striking difference between the sputtering yield of pure tungsten and helium pre-implanted tungsten $\left(\Phi=1.0 \times 10^{19} \mathrm{He} \cdot \mathrm{m}^{-2}\right.$ and 
(100) Surface

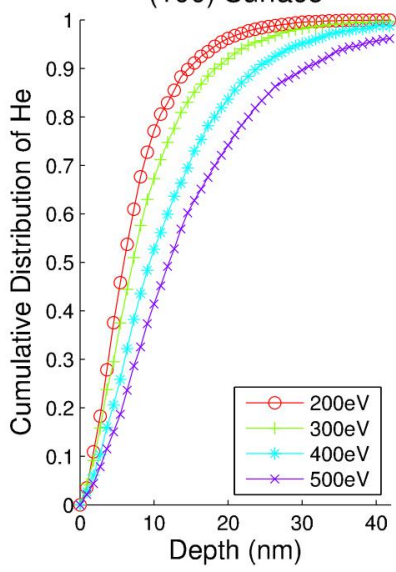

(100) Surface

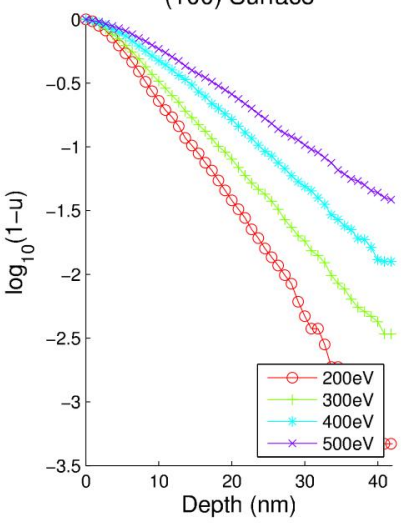

(100) Surface

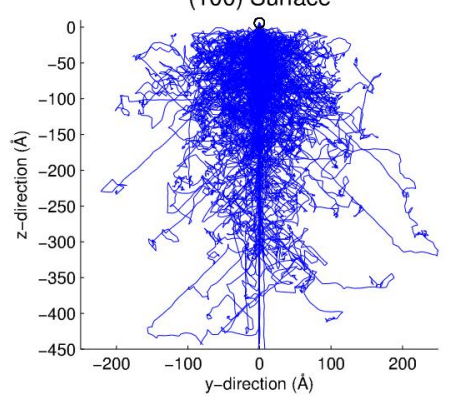

(110) Surface

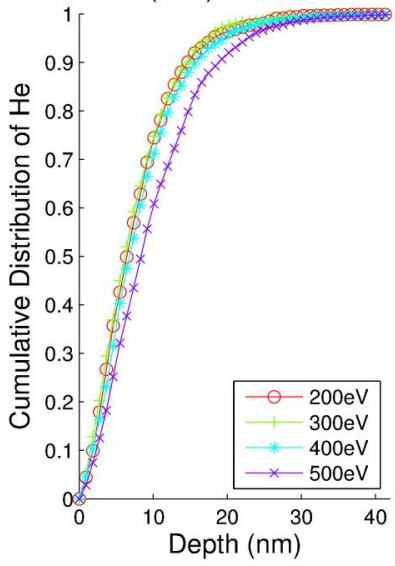

(110) Surface

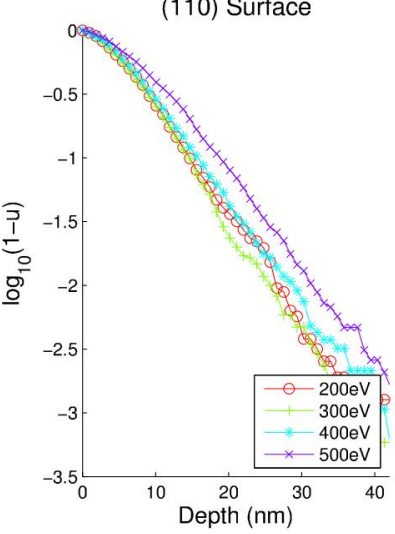

(110) Surface

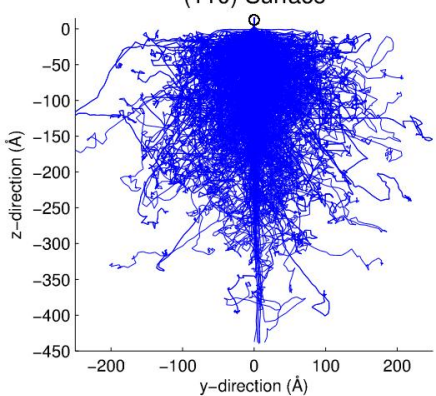

(111) Surface

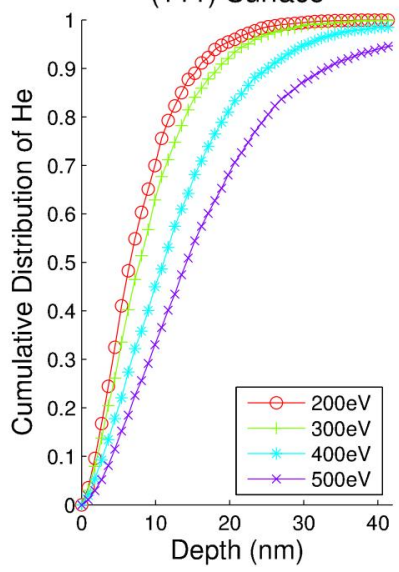

(111) Surface
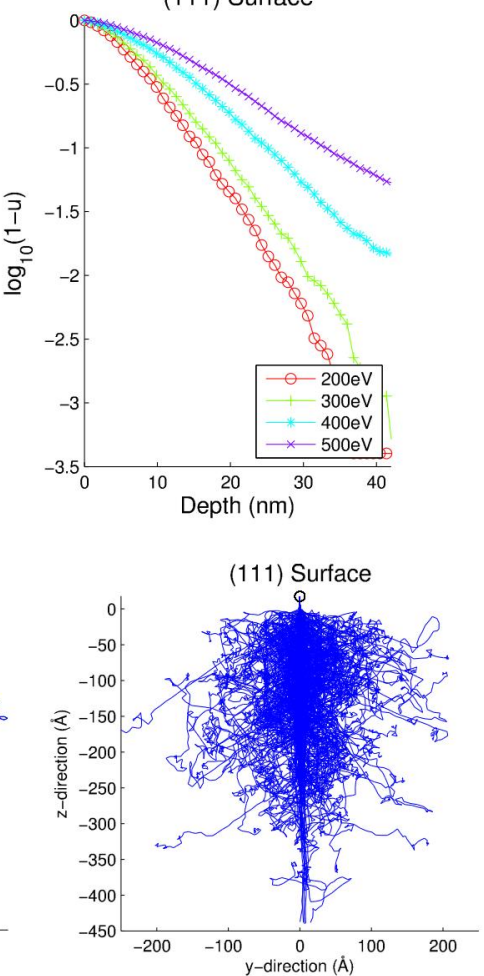

Figure 3: Cumulative depth distributions of helium embedded in tungsten as a function of incident energy for three surface orientations. The ordinate of the middle three plots is $y=\log _{10}(1-u)$, where $u$ is the ordinate of the corresponding upper plot. The bottom three plots shows a sample of $\sim 500$ trajectories at $500 \mathrm{eV}$ from a normalised starting position for each surface orientation. 


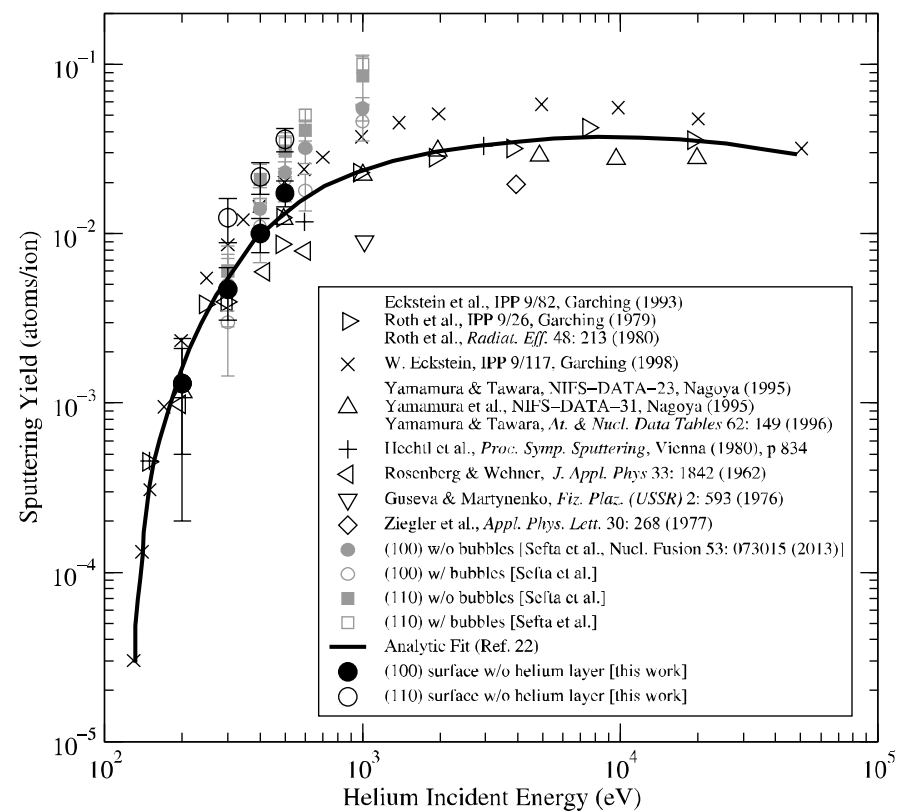

Figure 4: Sputtering yields as calculated in this paper and the work of Sefta [21] and select experimental data as collected in Ref. 24. Figure adapted from Refs. 21 and 24.

$\Phi=2.2 \times 10^{19} \mathrm{He} \cdot \mathrm{m}^{-2}$ ), within the uncertainty limits, as shown in Figure 5. It should be noted that $\mathrm{W}\{100\}$ and $\mathrm{W}\{110\}$ surfaces, unlike $\mathrm{W}\{111\}$, do not show a significant concentration of near-surface helium atoms. As such, we have no reason to expect sputtering on such surfaces to be any different than for the non-implanted surfaces.

\subsection{Helium Ejection}

For the case of pre-implanted tungsten, the number of helium atoms ejected was also tracked. The overwhelming majority of cases resulted in the incident helium atom being either embedded or reflected from the free surface without dislodging any existing tungsten or helium atoms, with similar reflection probabilities as in the pure tungsten scenarios. This results in a net change of $0-1$ helium atoms. However, in $\approx 1 \%$ of cases, a net loss of helium was observed. The majority of such cases resulted in a net loss of one helium atom, with a maximum loss of 16 helium atoms observed in once instance. Large amounts of helium release occur when the impinging helium atom impacts the surface 


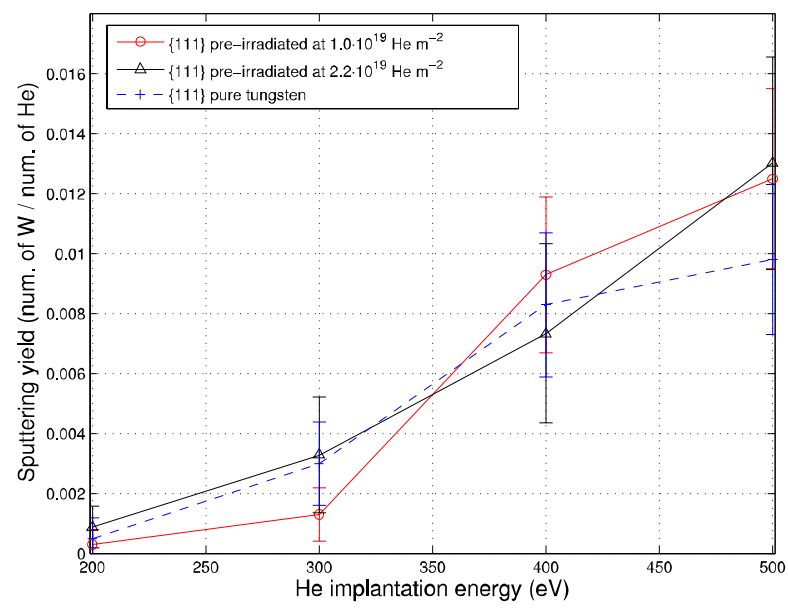

Figure 5: Comparison between sputtering yield of pure and pre-implanted tungsten as a function of helium incident energy for a $\left(\begin{array}{lll}1 & 1 & 1\end{array}\right)$ surface orientation at $933 \mathrm{~K}$. The pre-implanted "layer" of helium near the surface has very little, if any, effect on the sputtering yield.

directly over a near-surface bubble, locally increasing the kinetic energy and causing it to burst, as shown in Figure 6.

Ejection of more than one helium atom after a single bombardment was relatively rare, with the overall expected change in the number of helium atoms per incident helium atom $(\Delta \mathrm{He}$ /irradiation event) being around $0.6-0.7 /$ event (see Table 3).

The differences as a function of fluence appear small until one considers the trend with respect to the pure tungsten cases as shown in Table 2. It is clear that the fraction of ion bombardments that result in embedding at $500 \mathrm{eV}$ goes from $71 \%$ at no fluence to $70 \%$ at $\Phi=1.0 \times 10^{19} \mathrm{Hem}^{-2}$ to $69 \%$ at $\Phi=2.2 \times 10^{19} \mathrm{He} \mathrm{m}^{-2}$. A similar trend holds at all other energies: $400 \mathrm{eV}$, $69 \rightarrow 68 \rightarrow 66 ; 300 \mathrm{eV}, 67 \rightarrow 65 \rightarrow 64 ; 200 \mathrm{eV}, 63 \rightarrow 62 \rightarrow 61$. While not definitive (the differences are not substantially higher than the standard error), these trends suggest that the effective reflection coefficient rises slightly as a function of fluence. 


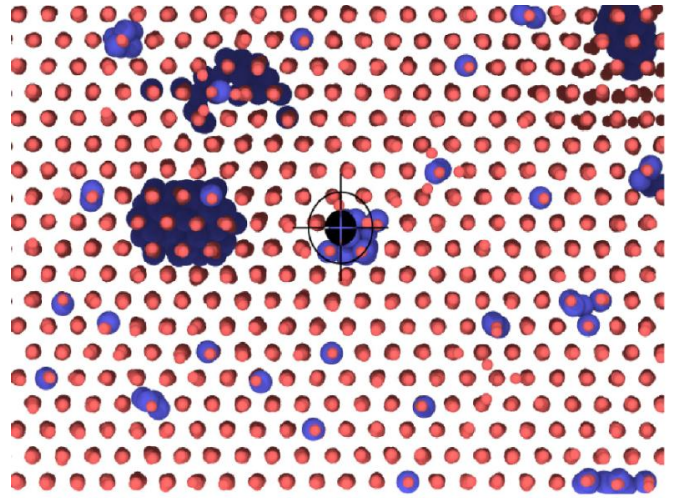

(a)

000000000000000008000 Tho 00000000

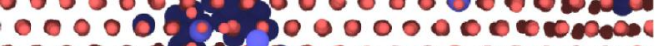
- o o o o 0000000000000000 . 0000000000000000000 - 000000000000000009 - 000. 0000000000006

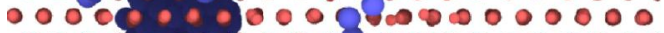

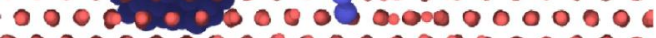
- 0 0 0 000000000000000 -0000000000000000000 - 00000000000000000000 0000000000000000000004 0000000000000000000000 00000000000000000006 0000000000000000000000 00000000000000000000 $-00000000000000000000$

(c)

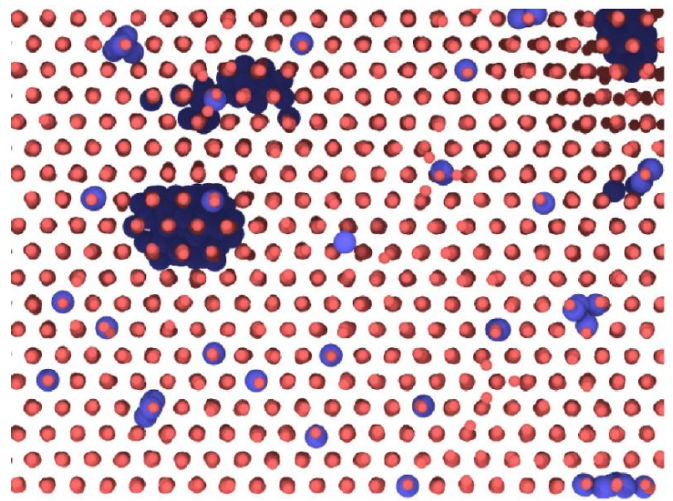

(e)

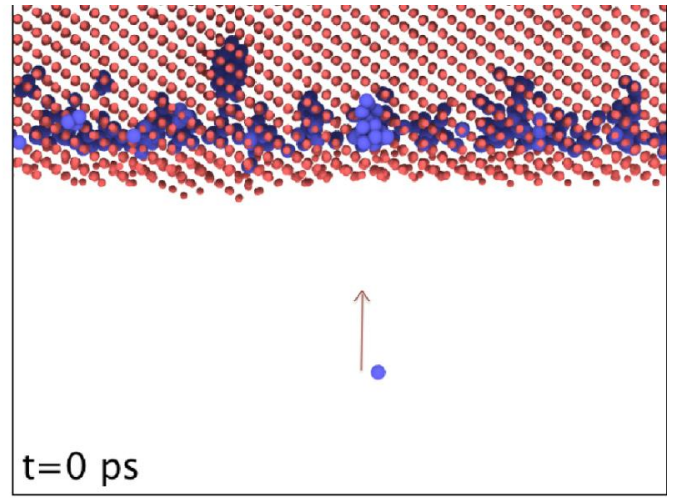

(b)

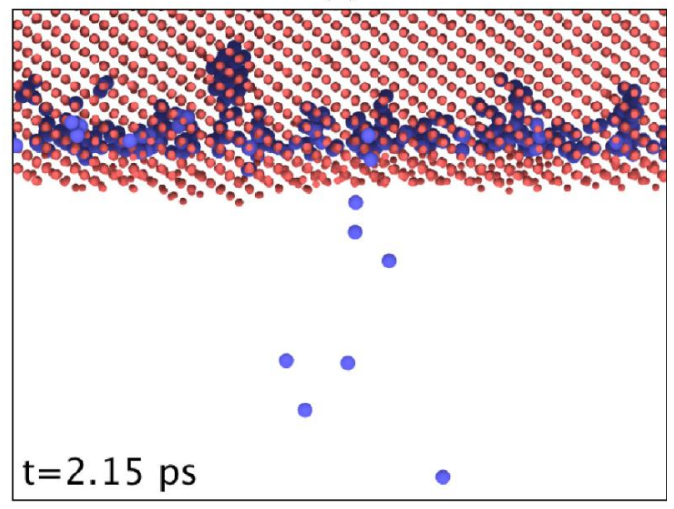

(d)

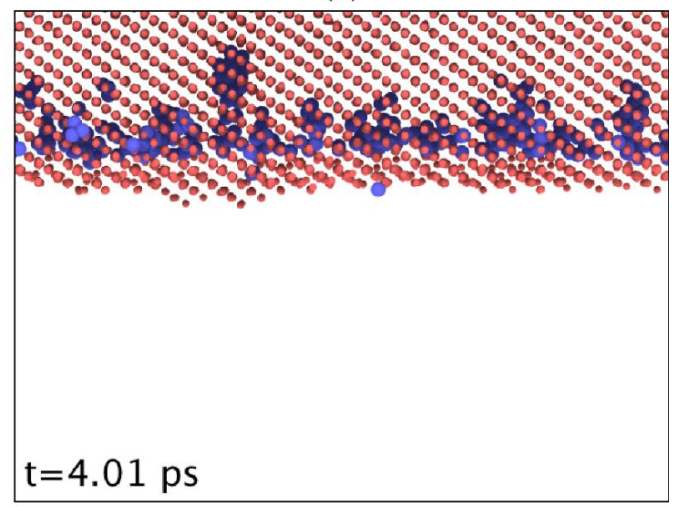

(f)

Figure 6: Demonstration of a near-surface helium bubble bursting as a result of an ion impinging on the surface direcly above it. Red (and smaller) atoms are tungsten, blue (and larger) are helium. (a),(b): Frontal and side snapshot prior to helium bombardment; (c),(d): Frontal and side snapshot after collision, showing sub-surface helium atoms ejected, (c),(f): Frontal and side snapshot showing removal of helium from a burst bubble. 
Table 3: Probabilities of net loss (negative $\Delta \mathrm{He}$ ), net gain (positive $\Delta \mathrm{He}$ ), and no net change (zero $\Delta \mathrm{He}$ ) of helium retention following a single helium-irradiation, and the expected value of $\Delta$ He per irradiation event, for two different fluences for a pre-implanted, (111)-oriented surface.

\begin{tabular}{|c|c|c|c|c|}
\hline \multicolumn{5}{|c|}{ Fluence: $1.0 \times 10^{19} \mathrm{He} \cdot \mathrm{m}^{-2}$} \\
\hline Energy $(\mathrm{eV})$ & Loss & No Change & Gain & $\langle\Delta \mathrm{He}\rangle$ \\
\hline 200 & $0.9 \%$ & $37.3 \%$ & $61.8 \%$ & 0.60 \\
\hline 300 & $0.9 \%$ & $33.9 \%$ & $65.2 \%$ & 0.64 \\
\hline 400 & $1.0 \%$ & $31.1 \%$ & $67.9 \%$ & 0.66 \\
\hline 500 & $1.1 \%$ & $29.2 \%$ & $69.7 \%$ & 0.68 \\
\hline
\end{tabular}

\begin{tabular}{lllll}
\multicolumn{5}{c}{ Fluence: $2.2 \times 10^{19} \mathrm{He} \cdot \mathrm{m}^{-2}$} \\
\hline Encrgy (cV) & Loss & No Changc & Gain & $\langle\Delta \mathrm{Hc}\rangle$ \\
\hline 200 & $1.2 \%$ & $37.8 \%$ & $61.0 \%$ & 0.59 \\
300 & $1.4 \%$ & $34.8 \%$ & $63.8 \%$ & 0.61 \\
400 & $1.3 \%$ & $32.4 \%$ & $66.3 \%$ & 0.64 \\
500 & $1.6 \%$ & $29.7 \%$ & $68.7 \%$ & 0.65 \\
\hline
\end{tabular}

\section{Conclusion}

The sputtering yiclds calculated from molecular dynamics simulations of pure and pre-implanted tungsten from helium irradiation under ITER-relevant conditions are not strongly dependent on the presence of relatively low concentrations of helium near the surface. We find that sputtering yields depend on surface orientation and incident helium energy, from a maximum of $0.036 \mathrm{~W} / \mathrm{He}$ for $\{110\}$ surfaces at $500 \mathrm{eV}$ incident energy to a minimum of $5 \times 10^{-4} \mathrm{~W} / \mathrm{He}$ for $\left\{\begin{array}{lll}1 & 1 & 1\end{array}\right\}$ surfaces at $200 \mathrm{eV}$ incident energy. The $\left\{\begin{array}{lll}1 & 1 & 0\end{array}\right\}$ orientations are the most prone to sputtering, followed by $\{100\}$ and lastly, $\{111\}$. We attribute these differences in surface orientation to the more closely-packed nature of $\{110\}$ surfaces compared to the other two orientations.

For a W(1 111$)$ surface pre-implanted with $100 \mathrm{eV}$ helium at a flux of $\Gamma=$ $7.9 \times 10^{25} \mathrm{He} \cdot \mathrm{m}^{-2} \mathrm{~s}^{-1}$ to either a fluence of $\Phi=1.0 \times 10^{19} \mathrm{He} \cdot \mathrm{m}^{-2}$ or $2.2 \times$ $10^{19} \mathrm{He} \cdot \mathrm{m}^{-2}$, the change in sputtering yields due to $200-500 \mathrm{eV}$ helium bombardment is negligible (within the $95 \%$ confidence intervals).

\section{Acknowledgements}

The authors express their thanks to Dr. Marie Backman at UT Knoxville for helpful discussions. This work used computing resources at the National Energy Research and Scientific Computing Facility (NERSC) at Lawrence Berkeley 
National Laboratory, which is supported by the Office of Science of the U. S. Department of Energy under contract DE-AC02-06CH11231; the IRIDIS High Performance Computing Facility and associated support services at the University of Southampton; and the Newton computing facility at the University of Tennessee. KDH and BDW acknowledge funding support from the Scientific Discovery through Advanced Computing (SciDAC) project on Plasma-Surface Interactions, funded the by the U. S. Department of Energy, Office of Science, Advanced Scientific Computing Research, and Fusion Energy Sciences under award DE-SC00-08875, and through the Plasma-Surface Interactions Science Center, funded the the U. S. Department of Energy, Office of Fusion Energy Sciences under award DE-SC00-02060. FF also acknowledges a travel grant from the Engineering and Physical Research Council's (EPSRC) Centre of Doctoral Training (CDT) in Fusion Energy Science and Technology.

Oak Ridge National Laboratory is managed by UT-Battelle, LLC for the U. S. Department of Energy under contract DE-AC05-00OR22725.

[1] G. Janeschitz, Plasma-wall interaction issues in ITER, J. Nucl. Mater. 290-293 (2001) 1-11.

[2] G. Federici, C. H. Skinner, J. N. Brooks, J. P. Coad, C. Grisolia, A. A. Haasz, A. Hassanein, V. Philipps, C. S. Pitcher, J. Roth, W. R. Wampler, D. G. Whyte, Plasma-material interactions in current tokamaks and their implications for next step fusion reactors, Nucl. Fusion 41 (12R) (2001) 1967-2136.

[3] H. Iwakiri, K. Yasunaga, K. Morishita, N. Yoshida, Microstruture evolution in tungsten during low-energy helium ion radiation, J. Nucl. Mater. 283 287 (2000) 1134-1138.

[4] M. Miyamoto, D. Nishijima, M. J. Baldwin, R. P. Doerner, Y. Ueda, K. Yasunaga, N. Yoshida, K. Ono, Microscopic damage of tungsten exposed to deuterium-helium mixture plasma in PISCES and its impacts on retention property, J. Nucl. Mater. 415 (2011) S657-S660. 
[5] S. Kajita, W. Sakaguuchi, N. Ohno, N. Yoshida, T. Saeki, Formation process of tungsten nanostructure by the exposure to helium plasma under fusion relevant plasma conditions, Nucl. Fusion 49 (2011) 095005.

[6] M. Yamagiwa, S. Kajita, N. Ohno, M. Takagi, N. Yoshida, R. Yoshihara, W. Sakaguchi, H. Kurishita, Helium bubble formation on tungsten in dependence of fabrication method, J. Nucl. Mater. 417 (2011) 499-503.

[7] F. Sefta, K. D. Hammond, N. Juslin, B. D. Wirth, Tungsten surface evolution by helium bubble nucleation, growth, and rupture, Nucl. Fusion 53 (7) (2013) 073015.

[8] K. D. Hammond, B. D. Wirth, Crystal orientation effects on helium ion depth distributions and adatom formation processes in plasma-facing tungsten, J. Appl. Phys.In press.

[9] A. Lasa, K. O. E. Henriksson, K. Nordlund, MD simulations of onset of tungsten fuzz formation under helium irradiation, Nucl. Instrum. Meth. Phys. Res. B 303 (2013) 156-161.

[10] B. L. Zhang, J. Wang, M. Li, Q. Hou, A molecular dynamics study of helium bubble formation and gas release near titanium surfaces, J. Nucl. Mater. 438 (1-3) (2013) 178-182.

[11] S. Plimpton, Fast parallel algorithms for short-range molecular dynamics, J. Comput. Phys. 117 (1) (1995) 1-19, http://lammps . sandia.gov/.

[12] M. W. Finnis, J. E. Sinclair, A simple empirical $N$-body potential for transition metals, Philos. Mag. A 50 (1) (1984) 45-55.

[13] G. J. Ackland, R. Thetford, An improved N-body semi-empirical model for b.c.c transition metals, Philos. Mag. A 56 (1) (1987) 15-30.

[14] N. Juslin, B. D. Wirth, Interatomic potentials for simulation of He bubble formation in W, J. Nucl. Mater. 432 (1-3) (2013) 61-66. 
[15] D. E. Beck, A new interatomic potential function for helium, Mol. Phys. 14 (4) (1968) 311-315.

[16] D. E. Beck, Erratum: A new interatomic potential function for helium, Mol. Phys. 15 (3) (1968) 332.

[17] K. Morishita, R. Sugano, B. D. Wirth, T. Diaz de la Rubia, Thermal stability of helium-vacancy clusters in iron, Nucl. Instrum. Meth. Phys. Res. B 202 (2003) 76-81.

[18] S. Nosé, A unified formulation of the constant temperature moleculardynamics methods, J. Chem. Phys. 81 (1) (1984) 511-519.

[19] W. G. Hoover, Canonical dynamics: Equilibrium phase-space distributions, Phys. Rev. A 31 (3) (1985) 1695-1697.

[20] G. J. Martyna, M. L. Klein, M. Tuckerman, Nosé-Hoover chains: The Canonical ensemble via continuous dynamics, J. Chem. Phys. 97 (4) (1992) 2635-2643.

[21] F. Sefta, N. Juslin, K. D. Hammond, B. D. Wirth, Molecular dynamics simulations on the effect of sub-surface helium bubbles on the sputtering yield of tungsten, J. Nucl. Mater. 438 (2013) S493-S496.

[22] C. E. Carlston, G. D. Magnuson, A. Comeaux, P. Mahadevan, Effect of elevated temperatures on sputtering yields, Phys. Rev. 138 (3A).

[23] V. S. Voitsenya, M. Balden, A. I. Belyaeva, V. K. Alimov, B. TyburskaPüschel, A. A. Galuza, A. A. Kasilov, I. V. Kolenov, V. G. Konovalov, O. O. Skoryk, S. I. Solodovchenko, Effect of sputtering on self-damaged recrystallized W mirror specimens, J. Nucl. Mater. 434 (1 3) (2013) 375 381.

[24] R. E. H. Clark (Ed.), Atomic and Plasma-Material Interaction Data for Fusion, Vol. 7B, International Atomic Energy Agency, Vienna, 2001, STI/PUB/023/APID/07/B. 
[25] V. Borovikov, A. F. Voter, X.-Z. Tang, Reflection and implantation of low energy helium with tungsten surfaces, J. Nucl. Mater. 447 (2014) 254-270. 